\title{
Astragaloside IV inhibits astrocyte senescence: implication in Parkinson's disease
}

\author{
Mei-Ling Xia ${ }^{1+}$, Xia-Hong Xie ${ }^{1+}$, Jian-Hua Ding ${ }^{2}$, Ren-Hong Du ${ }^{2^{*}}$ and Gang Hu ${ }^{1,2^{*}}$
}

\begin{abstract}
Background: Senescent astrocytes have been implicated in the aging brain and neurodegenerative disorders, including Parkinson's disease (PD). Astragaloside IV (AS-IV) is an antioxidant derivative from a traditional Chinese herbal medicine Astragalus membraneaceus Bunge and exerts anti-inflammatory and longevity effects and neuroprotective activities. However, its effect on astrocyte senescence in PD remains to be defined.
\end{abstract}

Methods: Long culture-induced replicative senescence model and lipopolysaccharide/1-methyl-4-phenylpyridinium $\left(\mathrm{LPS}_{\mathrm{MPP}}\right)^{+}$-induced premature senescence model and a mouse model of PD were used to investigate the effect of AS-IV on astrocyte senescence in vivo and in vitro. Immunocytochemistry, qPCR, subcellular fractionation, flow cytometric analyses, and immunohistochemistry were subsequently conducted to determine the effects of AS-IV on senescence markers.

Results: We found that AS-IV inhibited the astrocyte replicative senescence and LPS/MPP ${ }^{+}$-induced premature senescence, evidenced by decreased senescence-associated $\beta$-galactosidase activity and expression of senescence marker p16, and increased nuclear level of lamin B1, and reduced pro-inflammatory senescence-associated secretory phenotype. More importantly, we showed that AS-IV protected against the loss of dopamine neurons and behavioral deficits in the mouse model of PD, which companied by reduced accumulation of senescent astrocytes in substantia nigra compacta. Mechanistically, AS-IV promoted mitophagy, which reduced damaged mitochondria accumulation and mitochondrial reactive oxygen species generation and then contributed to the suppression of astrocyte senescence. The inhibition of autophagy abolished the suppressive effects of AS-IV on astrocyte senescence.

Conclusions: Our findings reveal that AS-IV prevents dopaminergic neurodegeneration in PD via inhibition of astrocyte senescence through promoting mitophagy and suggest that AS-IV is a promising therapeutic strategy for the treatment of age-associated neurodegenerative diseases such as PD.

Keywords: Astragaloside IV, Astrocyte, Cell senescence, Mitophagy, Parkinson's disease

\footnotetext{
* Correspondence: drh@njmu.edu.cn; ghu@njmu.edu.cn

${ }^{\dagger}$ Mei-Ling Xia and Xia-Hong Xie contributed equally to this work.

${ }^{2}$ Jiangsu Key Laboratory of Neurodegeneration, Department of

Pharmacology, Nanjing Medical University, 101 Longmian Avenue, Nanjing,

Jiangsu 211166, People's Republic of China

${ }^{1}$ Department of Pharmacology, School of Medicine and Life Sciences,

Nanjing University of Chinese Medicine, 138 Xianlin Avenue, Nanjing, Jiangsu

210023, People's Republic of China
}

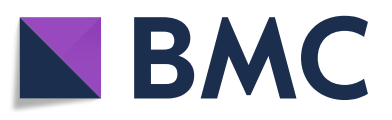

(C) The Author(s). 2020 Open Access This article is licensed under a Creative Commons Attribution 4.0 International License, which permits use, sharing, adaptation, distribution and reproduction in any medium or format, as long as you give appropriate credit to the original author(s) and the source, provide a link to the Creative Commons licence, and indicate if changes were made. The images or other third party material in this article are included in the article's Creative Commons licence, unless indicated otherwise in a credit line to the material. If material is not included in the article's Creative Commons licence and your intended use is not permitted by statutory regulation or exceeds the permitted use, you will need to obtain permission directly from the copyright holder. To view a copy of this licence, visit http://creativecommons.org/licenses/by/4.0/ The Creative Commons Public Domain Dedication waiver (http://creativecommons.org/publicdomain/zero/1.0/) applies to the data made available in this article, unless otherwise stated in a credit line to the data. 


\section{Background}

Parkinson's disease (PD), a common neurodegenerative disease, is characterized by the dopaminergic (DA) neuron death in the substantia nigra compacta (SNc) [1]. Although the mechanisms of PD have not still been clearly clarified, emerging evidence suggests that astrocytes have been involved in the pathogenesis of PD [2]. Astrocytes are the most populous glial subtype and are critical for brain function. They regulate ion balance and provide metabolic and neurotrophic support in the central nervous system (CNS). Therefore, the disruption of astrocyte biology may contribute to CNS dysfunction and pathology [3].

Aging is the most crucial risk factor for the development of PD [4]. Studies have shown that the annual incidence of PD increases exponentially with age. It is, therefore, possible that cell senescence contributes to its pathophysiology [5]. Cellular senescence is one of the essential mechanisms of aging and has been recognized as a central component of age-associated neurodegenerative disorders [6]. Senescent cells increase the secretion of proteases, pro-inflammatory cytokines, and chemokines, known as senescence-associated secretory phenotype (SASP), which strengthens cell senescence and spreads this phenotype to surrounding cells in a paracrine and autocrine way $[7,8]$. Increasing evidence indicates the cellular senescence that plays a profound role in the initiation and progression of neurodegenerative diseases such as PD and cognitive decline with aging [4] [9-11]. Therefore, targeting senescent astrocytes could represent a novel approach toward therapies for ageassociated neurodegenerative diseases [12].

Astragaloside IV (AS-IV) is purified from the Chinese medicinal herb, Astragalus membranaceus. AS-IV has been reported to exert anti-aging and immunomodulatory effects, and anti-inflammatory and anti-oxidative neuroprotective activities [13-16]. Previously, AS-IV was reported to reduce apoptosis and increase the resistance of dopamine neurons to neurotoxins in vitro [17]. Nevertheless, the effects of AS-IV on astrocyte function in PD have not yet been investigated. Given the importance of astrocyte senescence in PD, we hypothesize that AS-IV may inhibit astrocyte senescence from preventing DA neurodegeneration in the pathogenesis of PD. In the present study, we demonstrated that AS-IV significantly protected mice against neurodegeneration in the murine PD model through inhibiting senescent astrocytes. Further study revealed that AS-IV triggered mitophagy and then reduced the accumulation of dysfunctional mitochondria and mitochondrial reactive oxygen species (ROS) production, thereby inhibiting the astrocyte senescence. The findings obtained in this study may help direct clinical decisions regarding the use of AS-IV in age-associated neurodegenerative diseases such as PD.

\section{Methods}

\section{Experimental animals}

Mice were maintained and bred in the Animal Resource Centre of the Faculty of Medicine, Nanjing Medical University. Mice are free to access to food and water in a room with an ambient temperature of $22^{\circ} \mathrm{C} \pm 2{ }^{\circ} \mathrm{C}$ and a light/dark cycle of 12:12 h. All animal procedures were carried out in strict accordance with the guideline of the Institutional Animal Care and Use Committee of Nanjing Medical University.

\section{1-Methyl-4-phenyl-1,2,3,6-tetrahydropyridine (MPTP)- induced PD mouse model}

MPTP/ probenecid mouse model is used widely for preclinical in vivo assessment of anti-parkinsonian effects, due to its high reproducibility and disease-related pathological features. MPTP is first taken up by astrocytes and converted there to the toxic metabolite 1-methyl-4-phenylpyridinium $\left(\mathrm{MPP}^{+}\right)$by the $\mathrm{MAO}$ enzyme. $\mathrm{MPP}^{+}$is then released and selectively taken up to the dopaminergic nerve terminals by the dopamine transporters and causes the oxidative damage of dopaminergic neurons. Probenecid serves to elevate concentrations of MPTP in the brain by reducing renal elimination of the MPTP. Mice (male, aged 4-5 months old) were randomly divided into saline-treated group, MPTP-treated group, MPTP + AS-IV-treated group, and AS-IV alone treated group. In the MPTP-treated group, mice received subacute MPTP administration. MPTP (Sigma, St. Louis, MO, USA) was injected subcutaneously at $30 \mathrm{mg} \mathrm{kg}^{-1}$, followed by $250 \mathrm{mg} / \mathrm{kg}$ probenecid (Sigma, St. Louis, MO, USA) at intervals of $1 \mathrm{~h}$ for 5 consecutive days and left for 3 days. The mice received chronic MPTP administration as follows: mice were subcutaneously injected with $20 \mathrm{mg} / \mathrm{kg}$ MPTP and then intraperitoneally injected with $250 \mathrm{mg} / \mathrm{kg}$ probenecid at intervals of $1 \mathrm{~h}$ every 3.5 days for 5 weeks. Control mice were treated with saline and probenecid. In the MPTP + AS-IV-treated group, mice were administered with an intraperitoneal injection of $100 \mathrm{mg} \mathrm{kg}^{-1}$ AS-IV (Jingzhu Biotechnology, Nanjing, China) at 12-h intervals before MPTP injection. One week after the last injection, mice were anesthetized with $40 \mathrm{mg} \mathrm{kg}^{-1}$ sodium pentobarbital injection (Sigma, St. Louis, MO, USA). Brain tissues were homogenized for qPCR analysis and western blotting or were sectioned for immunohistochemistry.

\section{Behavioral analysis}

One week after the final injection of MPTP, the pole test, traction test, and rotarod test were performed. For the pole test, place the mouse head up on the top of a vertical wooden rough-surfaced pole (height $50 \mathrm{~cm}$, diameter $1 \mathrm{~cm}$ ). Each mouse was accustomed to the apparatus 1 day before testing. Record the total time ( $\mathrm{T}$ - 
total, until the mouse reached the floor with its four paws) and the turn time (T-turn, for the mouse to turn completely head downward). The mouse was allowed to descend three times, and the best performance for the T-turn and T-total was obtained. If the mouse did not turn entirely downwards, fell, or slipped down, the default value of $120 \mathrm{~s}$ was considered. For the traction test, place the mouse on a rope (diameter $5 \mathrm{~mm}$ ), and the scores range from 0 to 4 , based on its limb placements. The score was evaluated according to the following criteria: if four limbs seized the rope and moved to the side, the score was 4 , and if four limbs seized the rope but could not move to the side, the score was 3 . If the two or one front limbs seized the rope, the score was 2 or 1 , respectively. If the mouse slipped down, the score was 0 . In addition, the mice were allowed to hang upside down. For the rotarod test, mice were accustomed to the rotarod ( $5 \mathrm{~min}$ with a fixed speed of $12 \mathrm{rpm}$ ) for 3 days before the start of the experiment and then placed the mice on the rod and tested at $20 \mathrm{rpm}$ for $5 \mathrm{~min}$ and recorded the latency time which each animal could stay on the rod at test speed. The tester was blinded to all genotypes and treatment groups for each behavioral testing.

\section{Immunohistochemical analysis and immunofluorescence}

The immunostaining method was described in the previous publication [1]. To detect tyrosine hydroxylase $(\mathrm{TH})$, the sections were incubated with mouse monoclonal antibody against TH (T1299, 1:1000, Sigma, St Louis, MO, USA) and then for $1 \mathrm{~h}$ with anti-mouse IgG, HRP-linked secondary antibodies (7076, 1:5000, Cell Signaling Technology, USA). Immunoreactivity was visualized by incubation in the substrate-chromogen solution (DAB). Control staining was performed without primary antibodies. The total number of TH-positive neurons in the SNc was recorded stereologically using the optical fractionator method with the MicroBrightField Stereo-Investigator software (MicroBrightField, Williston, VT, USA).

For immunofluorescence staining, the sections were blocked with $10 \%$ bovine serum albumin (BSA) in Trisbuffered saline $(\mathrm{TBS})+0.05 \%$ Triton $\mathrm{X}-100$ for $1 \mathrm{~h}$ at $20{ }^{\circ} \mathrm{C}$. Next, the sections were incubated with the following primary antibodies at $4{ }^{\circ} \mathrm{C}$ overnight: anti-glial fibrillary acidic protein (GFAP, Millipore, MAB360; 1:800 dilution), anti-lamin B1 (Abcam, ab16048, 1:200 dilution), and anti-p16 (Santa Cruz Biotechnology, sc-56330, 1:200 dilution). After that, the sections were incubated with Alexa Fluor 488-conjugated or Alexa Fluor 555conjugated secondary antibody for $1 \mathrm{~h}$ at $20^{\circ} \mathrm{C}$. Then, the sections were washed and mounted onto glass slides. DAPI (Life Technologies, P36931) was used to visualize nuclei. Images were taken under a confocal microscope (Axiovert LSM510, Carl Zeiss Co., Germany).

\section{Primary astrocyte culture and treatment}

Astrocyte culture was described in the previous publication [18]. Briefly, the neonatal midbrain is trypsinized with $0.25 \%$ trypase (Amresco, Solon, $\mathrm{OH}$ ) at $37^{\circ} \mathrm{C}$ and dissociated, and the cells were plated on a poly-lysinetreated culture plates in Dulbecco's modified Eagle's medium (DMEM)/Ham's F12 medium containing 10\% fetal bovine serum (FBS, GIBCO, Gaithersburg, MD, USA). After $24 \mathrm{~h}$, the culture media were changed to complete medium. The purity of astrocytes was > 95\%, as determined with GFAP immunocytochemistry. Astrocytes were pretreated with AS-IV for $1 \mathrm{~h}$ before $\mathrm{MPP}^{+}$ (200 $\mu$ M, Sigma, St. Louis, MO, USA) or lipopolysaccharide (LPS, $500 \mathrm{ng} / \mathrm{ml}$, Sigma, St. Louis, MO, USA) stimulation for $24 \mathrm{~h}$.

To induce replicative cellular senescence, astrocytes were cultured for 40 days in vitro (DIV) and passaged every 4-5 days (passages 8-10) until their growth slowed to a point at which the cell number in the culture remained stationary (Fig. s1), and then treated with ASIV at the indicated concentration for 10 days. Control group astrocytes were cultured for 10 days in vitro and then treated with AS-IV at the indicated concentration for 10 days. For pharmacological measurement, autophagy inhibitor 3-MA (3 mM, Tocris, UK) was added to the cell culture medium $1 \mathrm{~h}$ before AS-IV treatment. The cell extracts were analyzed by qPCR, flow cytometry, and immunoblotting.

\section{$\beta$-Galactosidase staining}

Astrocytes seeded on chamber slides were washed with PBS and fixed in 4\% paraformaldehyde in PBS for 30 $\min$ at $20^{\circ} \mathrm{C}$. Astrocyte senescence was evaluated using $\beta$-galactosidase-based Senescence Cells Staining Kit (CS0030-1KT, Sigma-Aldrich, USA) according to the manufacturer's instructions. Astrocytes stained blue at pH 6 were observed by a light microscope. The astrocyte nuclei were stained with DAPI. The number of positively stained cells and the number of total cells number were counted. Positive cells are expressed as a percentage of total cell number. Data were obtained from three different cultures.

\section{Cell viability assay}

According to the manufacturer's instructions, Cell viability was measured by Cell Counting Kit-8 (CCK-8, Biotool, Houston, TX). Briefly, $4 \times 10^{4}$ cells per well were seeded in a 96-well plate and then were treated with ASIV $(0-200 \mu \mathrm{M})$ for 10 days. After that, $10 \mu \mathrm{l}$ of CCK-8 reagent was added to each well for $4 \mathrm{~h}$. Finally, the absorbance was measured at $450 \mathrm{~nm}$ using a multi-well spectrophotometer (Bio-Rad). The cell viability of ASIV-treated astrocytes was described as a percentage 
compared with control cells, and the cell viability of control cells was considered to be $100 \%$.

\section{Reverse transcription and quantitative real-time PCR}

Trizol reagent (Invitrogen, USA) was used to extract total RNA from SNc tissues and cultured astrocytes. Reverse transcription PCR was performed using a TAKARA PrimeScript RT reagent kit. Then, real-time PCR was measured using a QuantiTect SYBR Green PCR kit (Qiagen, Germany) with an ABI 7300 Fast Real-Time PCR System (Applied Biosystems, Foster City, CA, USA). GAPDH was used as an internal control for the real-time PCR amplification. The sequences of primers for real-time PCR analysis are as follows: GAPDH forward: CAAAAGGGTCATCTCC, reverse: CCCCAGCATCAAAGGTG. p16 ${ }^{\text {Ink4a }}$ forward: CGCTTC TCACCTCGCTTGT, reverse: TGACCAAGAACCTGCGA CC. IL-1 $\beta$ forward: TCATTGTGGCTGTGGAGAAG, reverse: AGGCCACAGGTATTTTGTCG. IL- $1 \alpha$ forward: AGTCAACTCATTGGCGCTTG, reverse: GAGAGAGAT GGTCAATGGCAGA. IL-6 forward: TCCTTCCTACCCCA ATTTCCA, reverse: GTCTTGGTCCTTAGCCACTCC. Cxcl-1 forward: TGCACCCAAACCGAAGTCAT, reverse: CTCCGTTACTTGGGGACACC. MMP-3 forward: GTTC TGGGCTATACGAGGGC, reverse: TTCTTCACGGTTGC AGGGAG. MMP-9 forward: CGACTTTTGTGGTCTTCC CC, reverse: AGCGGTACAAGTATGCCTCTG.

\section{Measurement of mitochondrial ROS and mitochondrial membrane potential}

Mitochondrial ROS and mitochondrial membrane potential methods were described in the previous publication [1]. Astrocytes were stained with MitoSOX $(2.5 \mu \mathrm{M}$, Invitrogen, USA) or with $\mathrm{JC} 1(10 \mu \mathrm{g} / \mathrm{ml}, \mathrm{T}-3168$, Invitrogen, USA) at $37^{\circ} \mathrm{C}$ for $30 \mathrm{~min}$. After washing cells with PBS twice, the cells were then resuspended in cold PBS containing 1\% FBS for flow cytometric analyses. The data were analyzed with the FCS Express software (Guava Easy Cyte ${ }^{\mathrm{rm}} 8$, Millipore, USA).

\section{Mitochondrial isolation}

Mitochondrial extraction kit (Solarbio, China) was used to isolate astrocyte mitochondria. Astrocytes were homogenized in ice-cold lysis buffer with a Dounce-type glass homogenizer and centrifuged at $600 \mathrm{~g}$ for $10 \mathrm{~min}$. The crude supernatant was then centrifuged for $10 \mathrm{~min}$ at $11,000 \mathrm{~g}$ to pellet the intact mitochondria. After that, the pellet was homogenized in $50 \mu \mathrm{l}$ lysis buffer with $1 \mu \mathrm{l}$ cocktail (Roche). Then, the homogenate was spun $\left(1000 \times g\right.$ for $10 \mathrm{~min}$ at $4{ }^{\circ} \mathrm{C}$ ) for three times to precipitate nuclei and tissue debris, yielding a supernatant enriched in mitochondria. Mitochondria from the supernatant were finally precipitated by centrifugation at $16,000 \times g$ at $4{ }^{\circ} \mathrm{C}$ for $15 \mathrm{~min}$.

\section{Western blotting analysis}

Cell lysates and SNc tissues were homogenized in RIPA lysis buffer, and protein concentration was determined by the Bradford assay (Bio-Rad, Hercules, CA, USA). A 30- $\mu$ g protein aliquot of each sample was separated using standard SDS-PAGE and transferred onto a PVDF membrane (Millipore, Bedford, MA). Immunoreactive bands were detected by enhanced chemiluminescence plus detection reagent (Pierce, Rockford, IL) and analyzed using the ImageQuant ${ }^{\text {tix }}$ LAS 4000 imaging system (GE Healthcare, Pittsburgh, PA, USA). The following primary antibodies were used: anti-TH (T1299, Sigma, St Louis, MO, USA), anti-p16 (sc-56330, Santa Cruz Biotechnology, USA), anti$\beta$-actin (BM0627, Boster, Pleasanton, CA, USA), anti-LC3 (3868, Cell Signaling Technology, USA), anti-p62 (23214, Cell Signaling Technology, USA), anti-COX IV (4844, Cell Signaling Technology, USA), anti-TOM20 (42406, Cell Signaling Technology, USA), anti-Parkin (ab77924, Abcam, USA), and anti-PINK1 (ab23707, Abcam, USA).

\section{Statistical analysis}

All data are expressed as means \pm SEM. The differences with different treatments were determined by one-way or two-way ANOVA, followed by the Tukey's post hoc test, and were considered as statistically significant at $p$ $<0.05$.

\section{Results}

AS-IV rescues MPTP-induced the loss of DA neurons and behavioral deficits in mice

The neuroprotective of AS-IV was evaluated in the MPTP model of PD. MPTP injection induces a significant loss of $\mathrm{TH}$ in the SNc, which is prevented by AS-IV (Fig. 1a, b). Western blot analysis reveals that MPTP-mediated reduction in TH protein expression is restored by AS-IV in the SNc (Fig. 1c, d). The traction test is utilized to measure muscle strength and equilibrium. There is a marked lower traction score in the MPTP-treated mice, which is prevented by ASIV (Fig. 1e). AS-IV also significantly reduces the behavioral deficits elicited by MPTP injected as measured by the accelerating rotarod test and the pole test (Fig. 1f, g) without affecting MPTP metabolism (Fig. s2). These data indicate that AS-IV rescues MPTP-induced PD like pathology in mice.

\section{AS-IV reduces the accumulation of senescent astrocytes in SNc of MPTP PD mice}

Increasing evidence indicates that senescent astrocytes play important roles in PD [11], prompting us to ask whether AS-IV could attenuate senescent astrocytes in SNc of MPTP model mice. Compared to control mice, the MPTP-injected mice displayed elevated expression of the senescence marker $\mathrm{p} 16^{\text {Ink4a }}$, and several SASP 


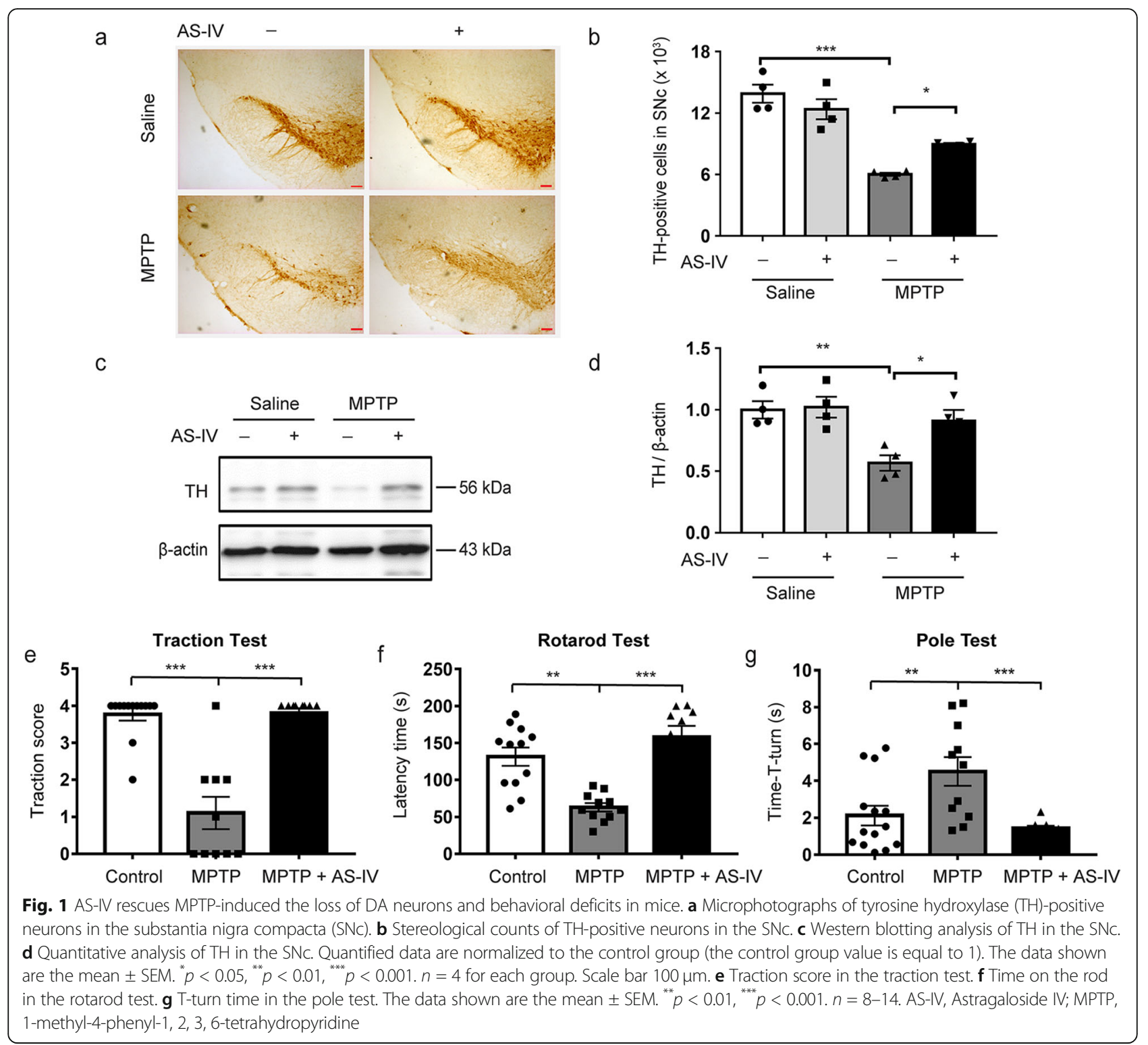

factors included the pro-inflammatory cytokines interleukin-6 (IL-6), and IL-1 $\beta$, which greatly decreased by the AS-IV treatment (Fig. $2 \mathrm{a}-\mathrm{c}$ ). We next determined whether AS-IV inhibited astrocyte senescence in PD. Remarkably, a significant population of senescent astrocytes overexpressing p16 is present in SNc of MPTP model mice, and the AS-IV treatment inhibited this MPTP-induced astrocyte senescence (Fig. 2d, f). In addition, a reduced nuclear level of lamin B1, an established senescence-associated marker, was found in astrocytes in SNc of MPTP model mice, and the AS-IV significantly suppressed the downregulation of nuclear lamin B1 (Fig. 2e, g). These results demonstrate that ASIV decreases the accumulation of senescent astrocytes in $\mathrm{SNc}$ of MPTP PD mice.

\section{AS-IV inhibits long-term culture-induced replicative senescence in astrocytes}

Next, we examined the direct effect of AS-IV on astrocyte senescence in vitro. To evaluate the role of AS-IV on replicative senescence, mouse astrocytes made senescent by in vitro culturing for 40 days, showed signs of a senescence phenotype, including elevated senescenceassociated $\beta$-galactosidase, and increased expression of senescence marker p16, which were indeed inhibited by AS-IV in a concentration-dependent manner without affecting cell survival (Fig. 3a-e). Since AS-IV induced a substantial suppressive effect at $50 \mu \mathrm{M}$ concentration, we used this concentration in the subsequent studies. Also, compared to nonsenescent astrocytes, senescent astrocytes displayed elevated expression of the several SASP 


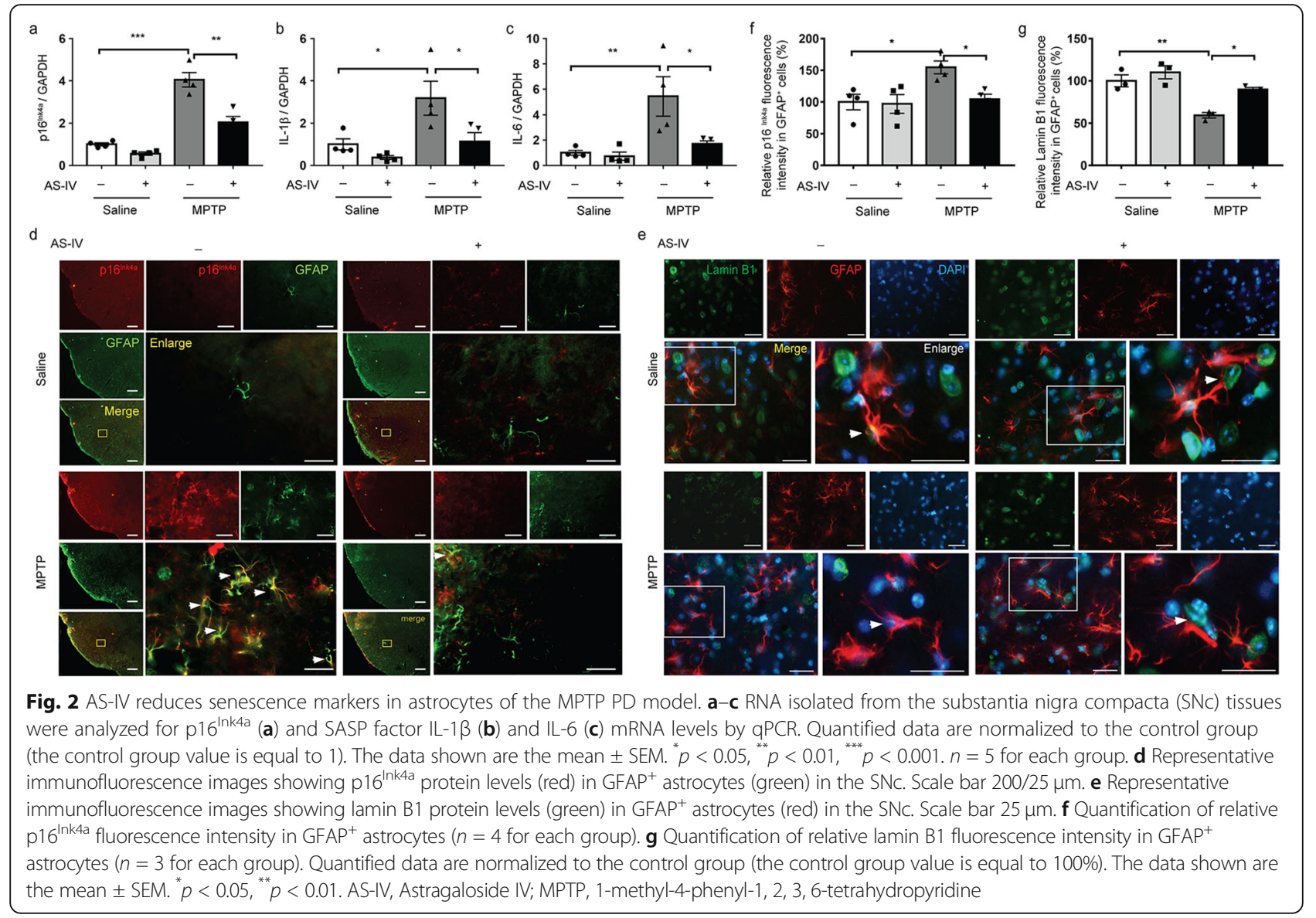

factors and reduced the nuclear level of lamin B1. AS-IV treatment inhibited the increase in SASP factors and a decrease in lamin B1 in senescent astrocytes (Fig. 3f-m). These findings indicate that AS-IV inhibits the replicative senescence of astrocytes.

\section{AS-IV suppresses $\mathrm{MPP}^{+} /$LPS-induced premature senescence of astrocytes}

We also assessed the ability of AS-IV on astrocytes to undergo senescence in response to $\mathrm{MPP}^{+}$, the active metabolite of MPTP significantly associated with PD. Cultured astrocytes exposed to $\mathrm{MPP}^{+}$displayed several hallmarks of senescence, including increased levels of p16 ${ }^{\text {Ink4a }}$ mRNA and protein (Fig. $4 \mathrm{a}-\mathrm{c}$ ), and elevated production of the pro-inflammatory IL-1 $\beta$, IL- $1 \alpha$, IL-6, Cxcl-1, MMP3, and MMP9, the prominent component of the SASP (Fig. 4d-i), and AS-IV suppressed $\mathrm{MPP}^{+}$-induced increase of $\mathrm{p} 16^{\text {Ink4a }}$ and the SASP production. As inflammation plays a critical role in senescence, we used a LPS-induced cell senescence model to verify the effects of AS-IV on astrocyte senescence. Astrocytes cultured with LPS for $24 \mathrm{~h}$ also displayed these phenotypes, such as increased $\mathrm{p} 16^{\mathrm{INK} 4 \mathrm{a}}$ mRNA and protein level and production of SASP. Significantly, all LPS-induced senescent phenotypes in astrocytes were abrogated by AS-IV treatment (Fig. $4 \mathrm{~h}-\mathrm{n}$ ). These data demonstrate that AS-IV inhibits $\mathrm{MPP}^{+} / \mathrm{LPS}$-induced astrocyte senescence.

\section{AS-IV promotes mitophagy and decreases the mitochondrial ROS production in astrocytes}

Increasing evidence indicates that autophagy has been reported to play crucial roles in cell senescence [19]. To investigate the mechanism by which AS-IV inhibits astrocyte senescence, we examined the levels of autophagy-associated proteins. As shown in Fig. 5a-c, the expression of microtubule-associated protein light chain 3 (LC3)-II was remarkably lower, while the level of p62 was significantly higher in senescent astrocytes than in nonsenescent astrocytes, as measured by immunoblotting, and this was markedly attenuated by AS-IV. Mitophagy is a specialized form of autophagy that plays an essential role in cell senescence. In addition, the expressions of mitophagy-related proteins that included PINK1 and Parkin were notably lower. In contrast, the level of TOM20 was higher in mitochondrial protein extracts from senescent astrocytes than nonsenescent astrocytes, indicating decreased mitophagy flux (Fig. 5d-f). AS-IV treatment 


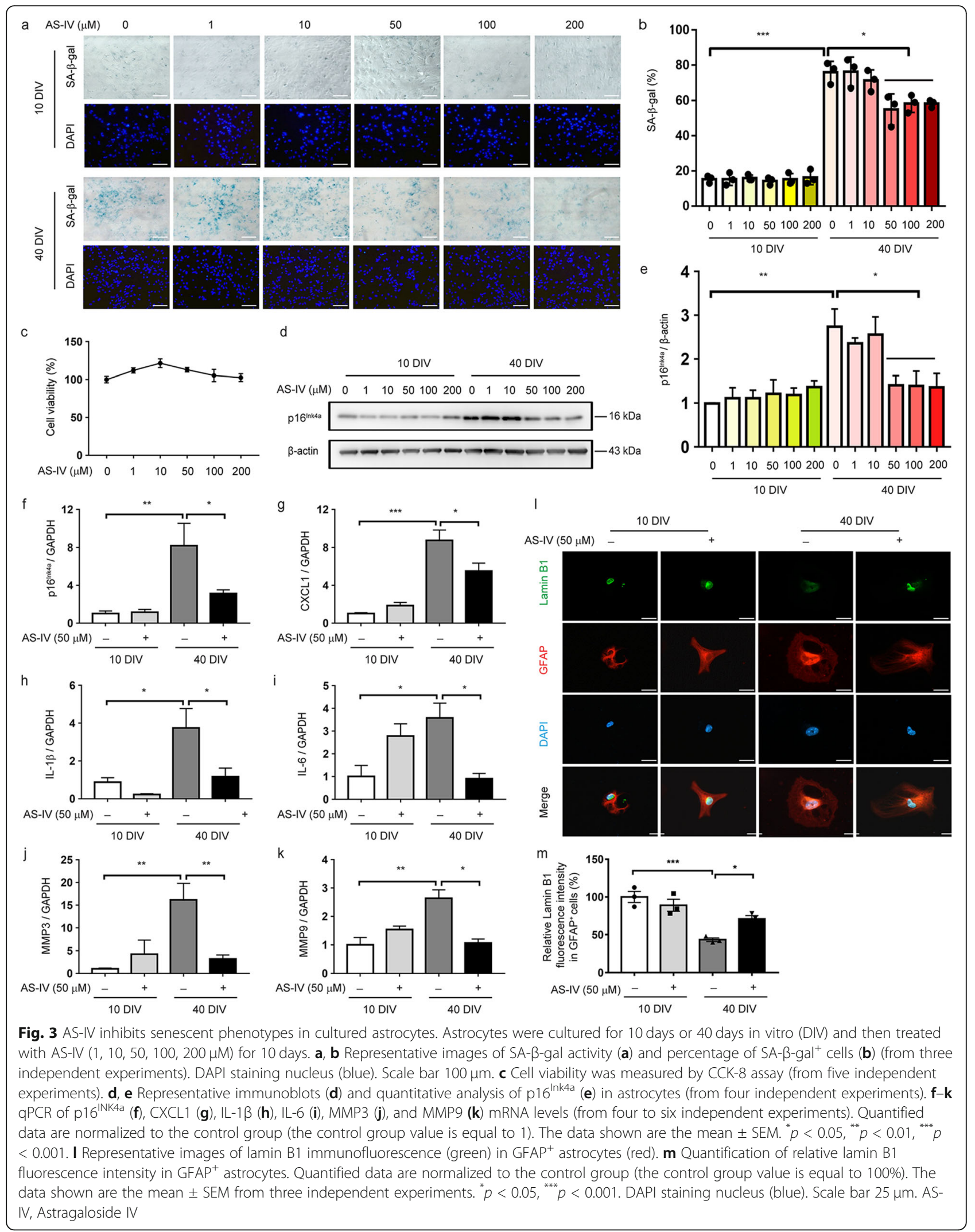




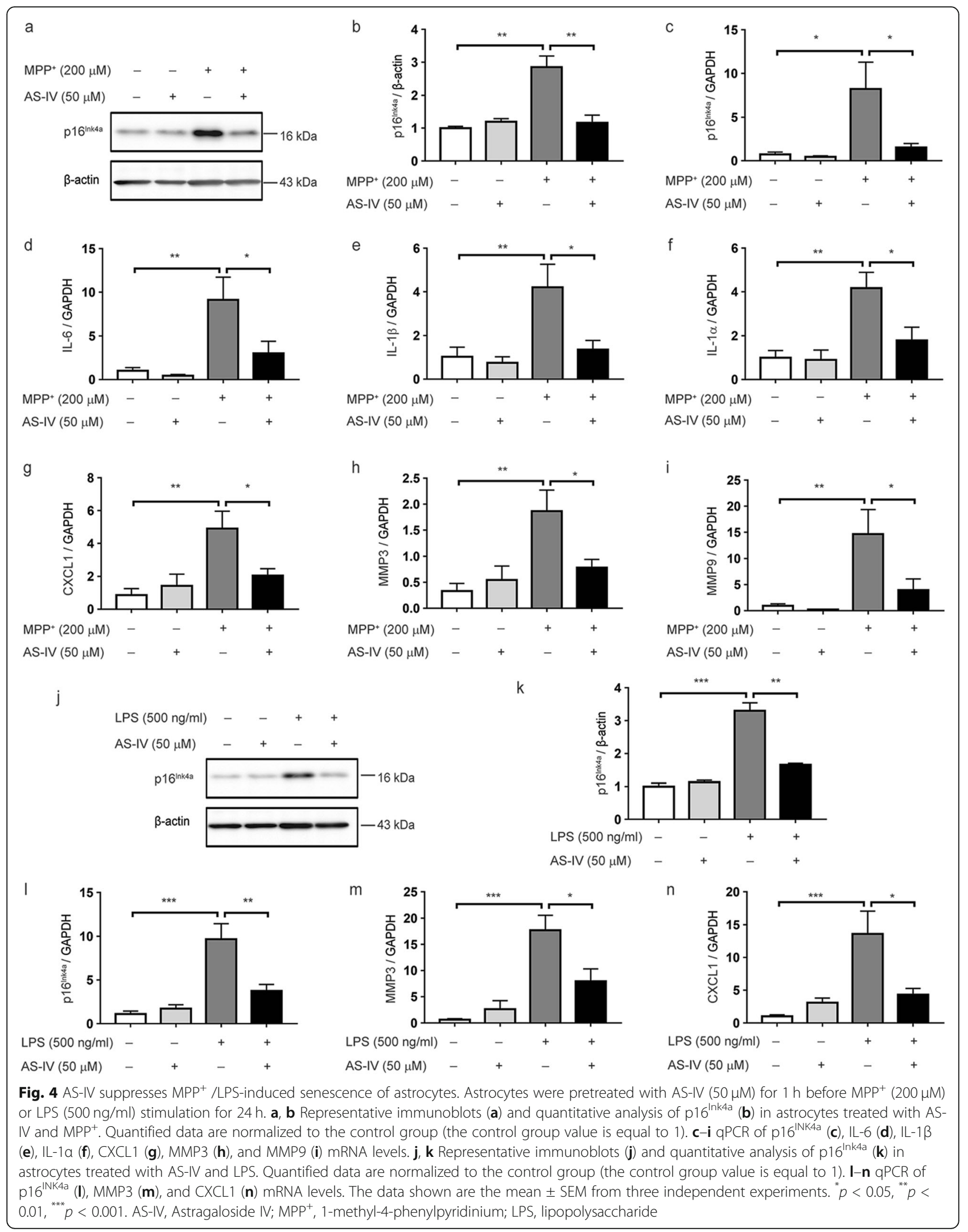



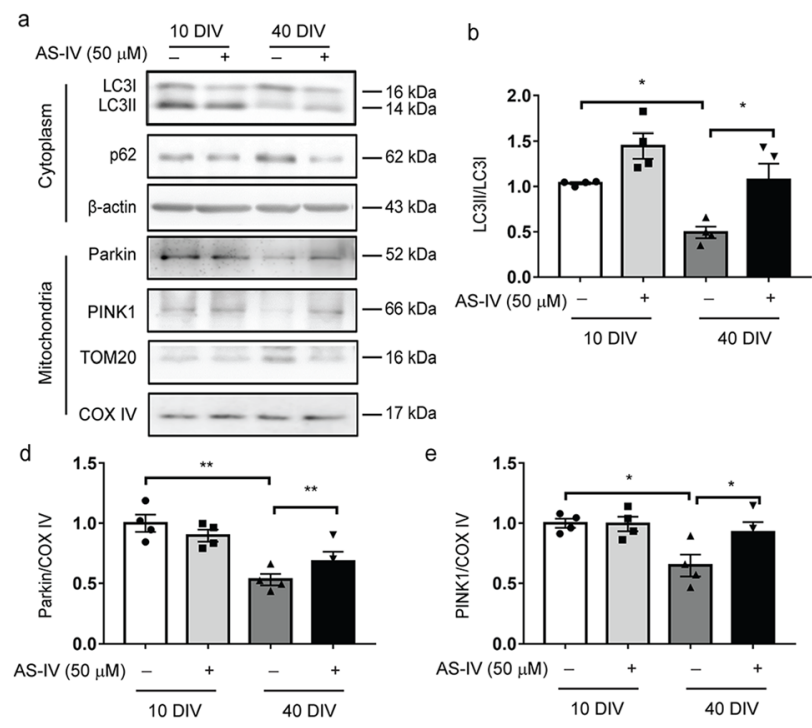

C
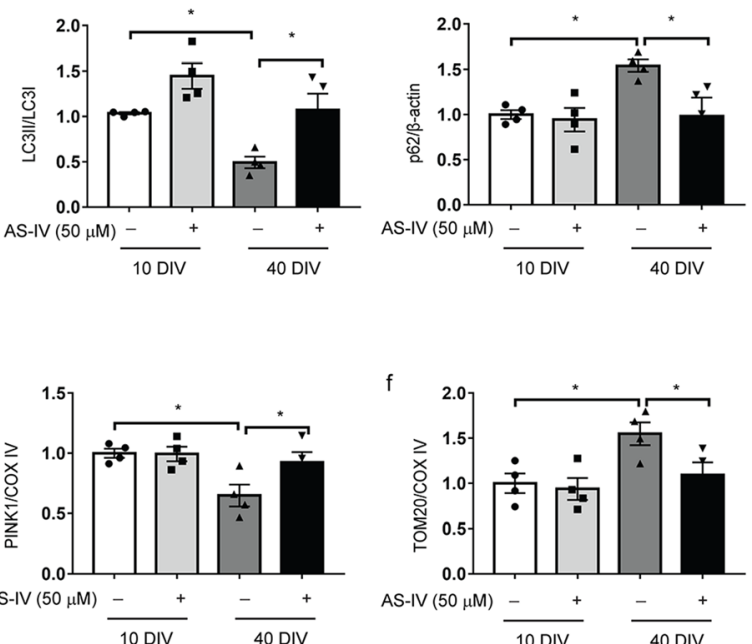

$\mathrm{h}$
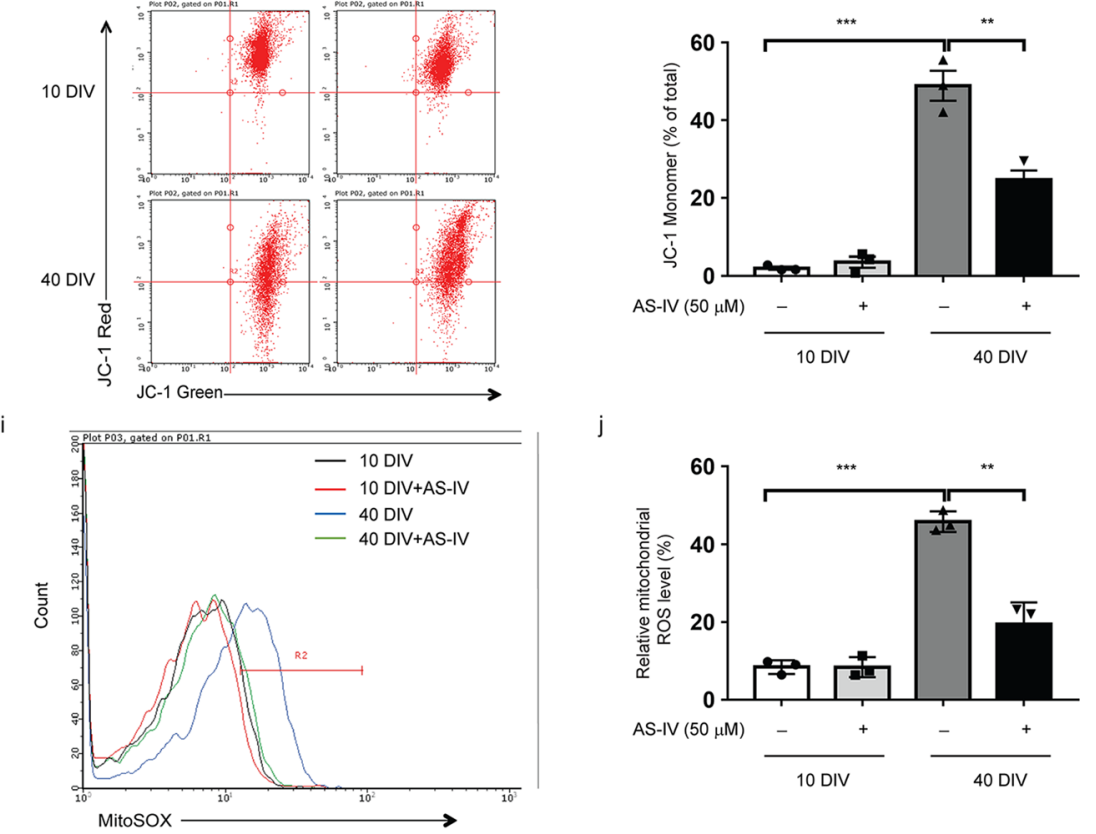

Fig. 5 AS-IV promotes mitophagy and decreases the mitochondrial ROS production in astrocytes. Astrocytes were cultured for 10 days or 40 days in vitro (DIV) and then treated with AS-IV (50 $\mu \mathrm{M})$ for 10 days. a-f Representative immunoblots (a) and quantitative analysis of p62 (b) and LC3 (c) in the cytoplasm and PINK1 (d), Parkin (e), and TOM20 (f) in the mitochondria of astrocytes. Quantified data are normalized to the control group (the control group value is equal to 1). $\mathbf{g}$ The scatter plot of the flow cytometry analysis shows the distribution of gating of JC-1 red-positive (aggregates) and JC-1 green-positive (monomers) cell population. $\mathbf{h}$ The bar graph shows the percentage of JC-1 monomer-positive cells. $\mathbf{i}$ Astrocytes was stained with MitoSOX and analyzed by flow cytometry. Representative dot plots are shown illustrating the gating of MitoSOXpositive (R2) populations. The percentages of MitoSOX-positive (R2) populations represent relative mitochondrial ROS levels. $\mathbf{j}$ Quantification of the mitochondrial ROS. The data shown are the mean \pm SEM from three independent experiments. ${ }^{*} p<0.05,{ }^{* *} p<0.01,{ }^{* * *} p<0.001$. AS-IV, Astragaloside IV

could restore the level of mitophagy in senescent astrocytes. More importantly, AS-IV improved the mitochondrial membrane potential, as measured using a fluorescence probe JC-1 assay system (Fig. $5 \mathrm{~g}$, h), and reduced robust mitochondrial ROS production in senescent astrocytes, as measured by mitochondrial superoxide indicator MitoSOX (Fig. 5i, j). These results suggest that AS-IV improves mitochondrial function and decreases mitochondrial ROS generation via promoting mitophagy in senescent astrocytes. 
Inhibition of autophagy reverses the suppressive effect of AS-IV on astrocyte senescence

To test whether autophagy is one of the main mechanisms of AS-IV-mediated astrocyte senescence inhibition, we used the autophagy inhibitor 3-MA to determine if inhibition of autophagy could abolish the suppressive effect of AS-IV on astrocyte senescence. We found that the inhibitory effects of AS-IV on all senescent phenotypes, including downregulated expression of p16 ${ }^{\text {Ink4a }}$ (Fig. 6a, b), decreased SA- $\beta$-gal activity (Fig. 6c, d), and reduced SASP (Fig. 6e-g) in astrocytes, were significantly blocked by 3-MA. These data indicate that AS-IV inhibits astrocyte senescence through promoting autophagy.

\section{Discussion}

The most critical finding presented here is that the small molecule AS-IV inhibits astrocyte senescence. Cellular senescence, an age-associated phenomenon, was first discovered in vitro by extensive culturing of fibroblasts [20]. Emerging evidence displayed that astrocytes from Alzheimer's disease patients showed increased expression of the senescence markers p21 and p16. Besides, it was demonstrated that astrocytes exhibited eventual growth arrest at late passages of in vitro culturing compared to early passages, indicating replicative senescence from primary astrocytes [21, 22]. Astrocytes have also been documented to undergo stress-induced premature senescence [23-25]. Therefore, astrocytes have been displayed to undergo cellular senescence in vitro due to a variety of factors such as replicative lifespan or external stressors. Resveratrol and a p38MAPK inhibitor could reverse the senescent phenotypes, indicating that senescence has been therapeutically targeted [26, 27]. In the present study, we have found that small molecule AS-IV inhibits senescent astrocyte phenotypes that include a

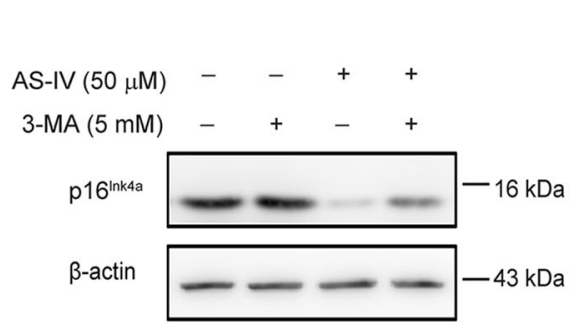

b

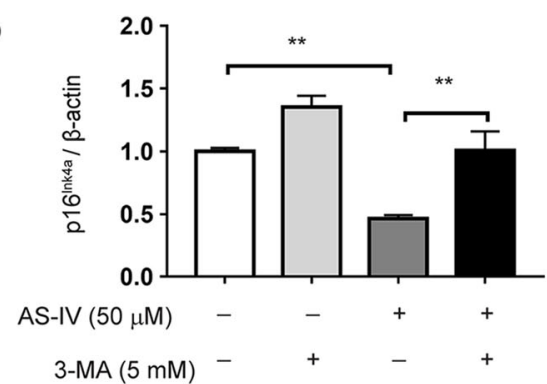

d

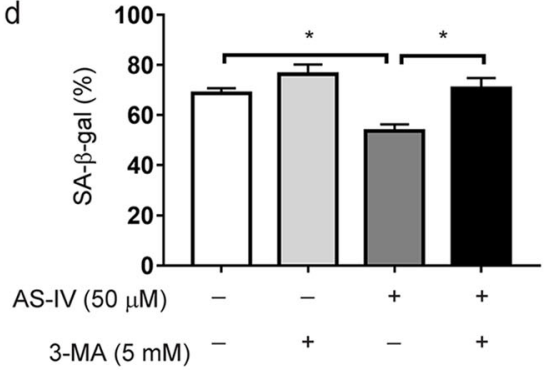

e

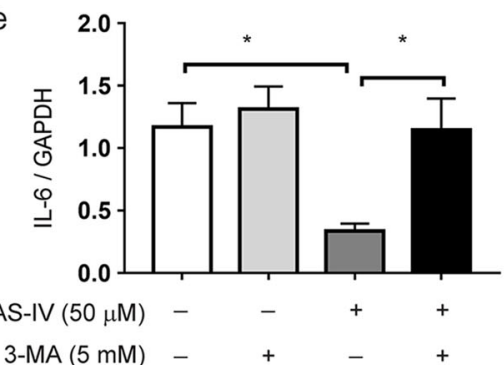

f

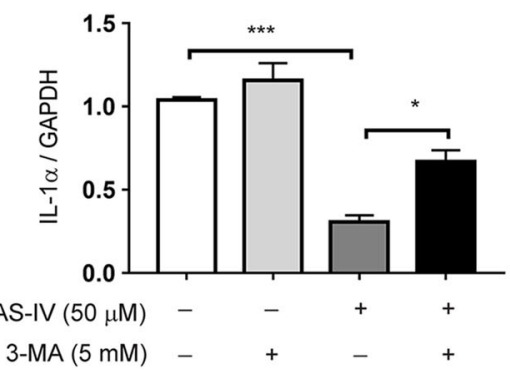

g

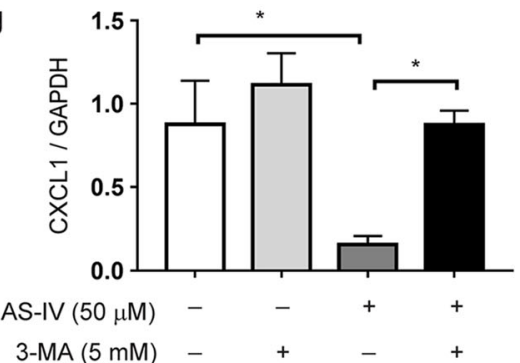

Fig. 6 Inhibition of autophagy reverses the suppressive effect of AS-IV on astrocyte senescence. Astrocytes (40 days) were treated with 3-MA (3 $\mathrm{mM})$ for $1 \mathrm{~h}$ before AS-IV $(50 \mu \mathrm{M})$ treatment for 10 days. $\mathbf{a}$, b Representative immunoblots (a) and quantitative analysis of p16 ${ }^{\text {Ink4a }}$ (b) in astrocytes. Quantified data are normalized to the control group (the control group value is equal to 1). $\mathbf{c}$, $\mathbf{d}$ Representative images of SA- $\beta$-gal activity (c) and the percentage of SA- $\beta$-gal ${ }^{+}$cells (d). DAPI staining nucleus (blue). Scale bar $100 \mu \mathrm{m}$. e-g qPCR of IL-6 (e), IL-1a (f), and CXCL1 (g) mRNA levels. The data shown are the mean \pm SEM from three to five independent experiments. ${ }^{*} p<0.05,{ }^{* *} p<0.01$, ${ }^{* * *} p<0.001$. AS-IV, Astragaloside IV 


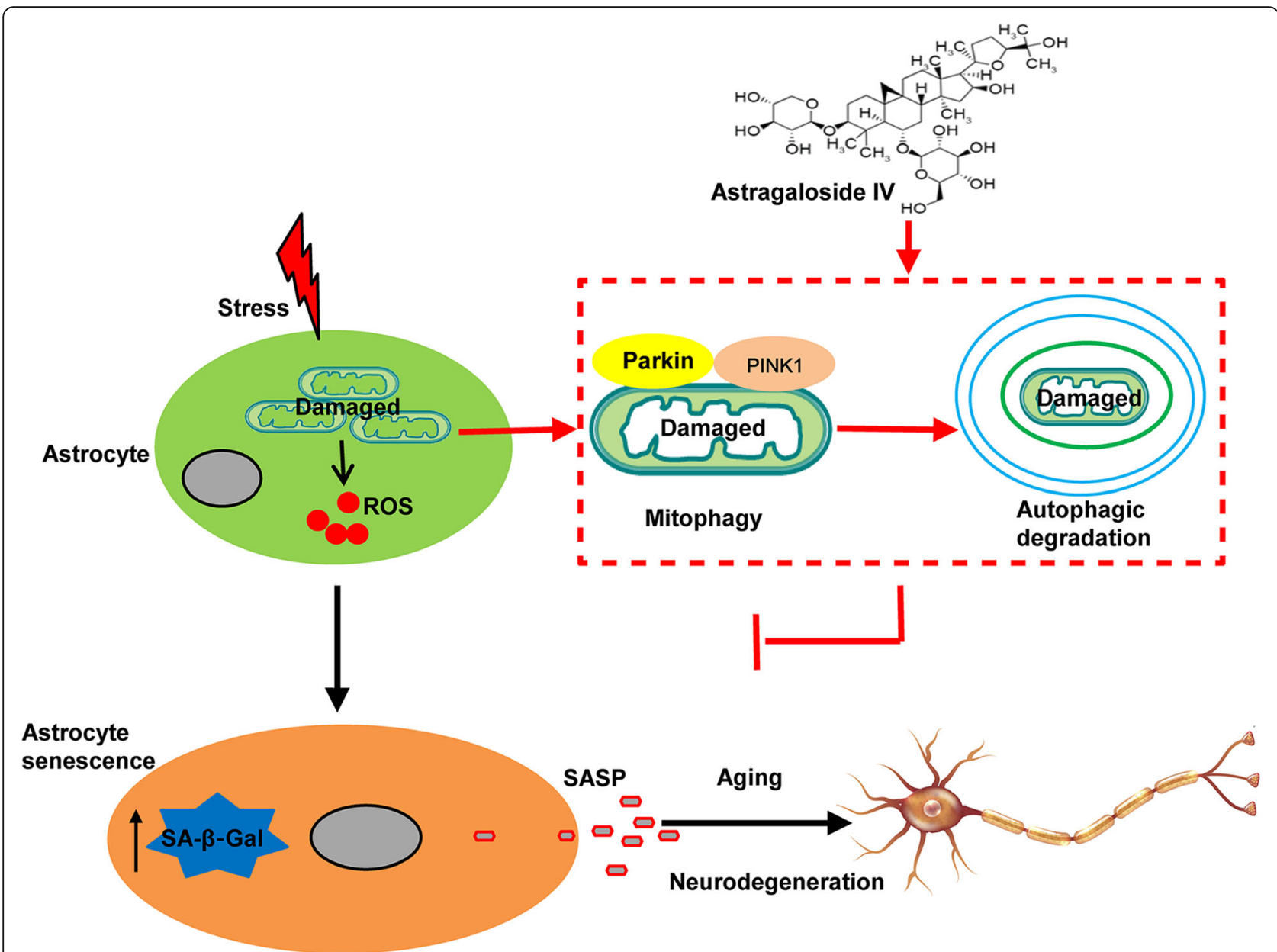

Fig. 7 Proposed model depicting AS-IV functions as an autophagy promoter to degrade damaged mitochondria and reduce ROS production, which inhibits astrocyte senescence and, consequently, the neurodegenerative process

increased SA- $\beta$-gal activity, elevated expression of the senescence marker $\mathrm{p} 16^{\text {Ink4a }}$, upregulated several SASP factors, and reduced nuclear level of lamin B1, in both extended culture-induced replicative senescence model and $\mathrm{MPP}^{+} / \mathrm{LPS}$-induced premature senescence model. More importantly, we have also shown that AS-IV treatment reduces the expression of $\mathrm{p} 16^{\mathrm{Ink} 4 \mathrm{a}}$ and increases the nuclear level of lamin B1 in astrocytes in the MPTPinduced PD model. These data indicate that small molecule AS-IV could suppress astrocyte senescence in vitro and PD model mice.

Our study further reveals the molecular mechanism underlying the suppressive effects of AS-IV on astrocyte senescence. Metabolic inflammation, oxidative stress, and mitochondrial dysfunction are believed to be involved in cell senescence $[28,29]$. Emerging evidence has revealed a positive correlation between activation of autophagy and longevity [30, 31]. Autophagy is a catabolic degradation system used to degrade and recycle the unnecessary or damaged components of a cell [32].
Increasing evidence shows that autophagy defective is implicated in aging and age-related pathologies. Thus, pharmacologically stimulated autophagy may ameliorate the symptoms of age-associated phenotypes [33, 34]. Mitophagy is a specialized form of autophagy that degraded the damaged and dysfunctional mitochondria [35]. A reduction in mitophagy leads to damaged mitochondria accumulation and oxidative stress, which contribute to cell senescence [36]. In the present study, we have observed the defective mitophagy in senescent astrocytes that included reduced LC3-II expression and elevated p62 expression, decreased PINK1 and Parkin levels, and increased TOM20 expression in mitochondria. The defective mitophagy in senescent astrocytes was reversed by treatment with AS-IV. As a consequence, AS-IV decreases the accumulation of damaged mitochondria and mitochondrial ROS generation in senescent astrocytes. Importantly, inhibition of autophagy abolishes the inhibitory effects of AS-IV on senescent astrocyte phenotypes. These findings indicate that AS-IV 
inhibits astrocyte senescence by promoting mitophagy. However, it should be further confirmed whether inhibition of autophagy in vivo reverses the suppressive effects of AS-IV on astrocyte senescence and DA neuron degeneration in the MPTP PD mouse model.

Growing evidence indicates that astrocyte senescence plays a vital role in the pathogenesis of PD [37, 38]. Astrocytes provide structural, metabolic, and trophic support to neurons $[39,40]$. When astrocytes are dysfunctional, they can impair neuronal function and lead to age-associated neurodegeneration in vivo [2, 41, 42]. Factors secreted by senescent astrocytes had detrimental effects on both cultured human DA neurons and neural progenitor cells $[43,44]$. Importantly, it was demonstrated that the levels of senescence markers in astrocytes from PD patients were significantly higher compared to control. Paraquat, an environmental insult significantly associated with PD, caused astrocyte senescence in vitro and in mice. In addition, depletion of senescent astrocytes alleviated PD-like neuropathological phenotypes [11]. In the present study, we have shown that MPTP PD mice had high levels of astrocytes positive for senescence markers, which companied by increased the loss of DA neurons and behavior deficits. AS-IV inhibited astrocyte senescence both in vitro and in mice and rescued DA neuron loss and behavioral deficits in the mouse model of PD. Therefore, it is reasonable to conclude that AS-IV promotes mitophagy, and this enhanced mitophagy decreases the damaged mitochondria accumulation and reduces mitochondrial ROS production in astrocytes, which contributes to the inhibition of astrocyte senescence and SASP factors secretion, thereby attenuating PD pathology (Fig. 7).

\section{Conclusions}

Our findings reveal that AS-IV prevents DA neurodegeneration in PD via inhibition of astrocyte senescence through promoting mitophagy and suggest that AS-IV is a promising therapeutic strategy for the treatment of age-associated neurodegenerative disease such as PD.

\section{Supplementary information}

Supplementary information accompanies this paper at https://doi.org/10. 1186/s12974-020-01791-8.

Additional file 1: Figure S1 and S2.

\footnotetext{
Abbreviations

PD: Parkinson's disease; AS-IV: Astragaloside IV; LPS: Lipopolysaccharide; $\mathrm{MPP}^{+}$: 1-Methyl-4-phenylpyridinium; DA: Dopaminergic; SNc: Substantia nigra compacta; CNS: Central nervous system; MPTP: 1-Methyl-4-phenyl-1,2,3,6tetrahydropyridine; TH: Tyrosine hydroxylase; GFAP: Glial fibrillary acidic protein; SASP: Senescence-associated secretory phenotype; ROS: Reactive oxygen species; IL-6: Interleukin-6; LC3: Microtubule-associated protein light chain 3
}

\section{Acknowledgements}

Not applicable

\section{Authors' contributions}

GH conceived the study and revised the paper. RHD designed the study and wrote the paper. MLX, XHX, and JHD performed the experiments and analyzed the data. All authors read and approved the final manuscript.

\section{Funding}

This work was supported by the grants from the Drug Innovation Major Project (2018ZX09711001-003-007), the National Natural Science Foundation of China (No. 81630099), and the Natural Science Foundation of the Jiangsu Higher Education Institutions of China (No. 19KJA310004).

\section{Availability of data and materials}

All data generated and/or analyzed during this study are included in this article.

\section{Ethics approval and consent to participate}

The animals used in our study were treated in accordance with protocols approved by the Institutional Animal Care and Use Committee of Nanjing Medical University.

\section{Consent for publication}

Not applicable

\section{Competing interests}

The authors declare that they have no conflict of interest.

Received: 2 November 2019 Accepted: 27 March 2020

Published online: 06 April 2020

\section{References}

1. Hu ZL, Sun T, Lu M, Ding JH, Du RH, Hu G. Kir6.1/K-ATP channel on astrocytes protects against dopaminergic neurodegeneration in the MPTP mouse model of Parkinson's disease via promoting mitophagy. Brain Behav Immun. 2019;81:509-22.

2. Liddelow SA, Guttenplan KA, Clarke LE, Bennett FC, Bohlen CJ, Schirmer L, Bennett ML, Munch AE, Chung WS, Peterson TC, et al. Neurotoxic reactive astrocytes are induced by activated microglia. Nature. 2017;541:481-7.

3. Trujillo-Estrada L, Gomez-Arboledas A, Forner S, Martini AC, Gutierrez A, Baglietto-Vargas D, La Ferla FM. Astrocytes: from the physiology to the disease. Curr Alzheimer Res. 2019;16:675-98.

4. Cohen J, Torres C. Astrocyte senescence: evidence and significance. Aging Cell. 2019;18:e12937.

5. Naylor RM, Baker DJ, van Deursen JM. Senescent cells: a novel therapeutic target for aging and age-related diseases. Clin Pharmacol Ther. 2013;93:10516.

6. Mendelsohn AR, Larrick JW. Cellular senescence as the key intermediate in tau-mediated neurodegeneration. Rejuvenation Res. 2018;21:572-9.

7. Maciel-Baron LA, Moreno-Blas D, Morales-Rosales SL, Gonzalez-Puertos VY, Lopez-Diazguerrero NE, Torres C, Castro-Obregon S, Konigsberg M. Cellular senescence, neurological function, and redox state. Antioxid Redox Signal. 2018;28:1704-23.

8. Santoro A, Spinelli CC, Martucciello S, Nori SL, Capunzo M, Puca AA, Ciaglia E. Innate immunity and cellular senescence: the good and the bad in the developmental and aged brain. J Leukoc Biol. 2018;103:509-24.

9. Musi N, Valentine JM, Sickora KR, Baeuerle E, Thompson CS, Shen Q, Orr ME. Tau protein aggregation is associated with cellular senescence in the brain. Aging Cell. 2018;17:e12840

10. Bussian TJ, Aziz A, Meyer CF, Swenson BL, van Deursen JM, Baker DJ, Clearance of senescent glial cells prevents tau-dependent pat hology and cognitive decline. Nature. 2018;562:578-82.

11. Chinta SJ, Woods G, Demaria M, Rane A, Zou Y, McQuade A, Rajagopalan S, Limbad C, Madden DT, Campisi J, Andersen JK. Cellular senescence is induced by the environmental neurotoxin paraquat and contributes to neuropathology linked to Parkinson's disease. Cell Rep. 2018;22:930-40.

12. Scott KM, Williams-Gray CH. Targeting aged astrocytes may be a new therapeutic strategy in Parkinson's disease. Mov Disord. 2018;33:758-9.

13. Wen W, Chen J, Ding L, Luo X, Zheng X, Dai Q, Gu Q, Liu C, Liang M, Guo X, et al. Astragaloside exerts anti-photoaging effects in UVB-induced 
premature senescence of rat dermal fibroblasts through enhanced autophagy. Arch Biochem Biophys. 2018;657:31-40.

14. Jiang M, Ni J, Cao Y, Xing X, Wu Q, Fan G. Astragaloside IV Attenuates myocardial ischemia-reperfusion injury from oxidative stress by regulating succinate, lysophospholipid metabolism, and ROS scavenging system. Oxidative Med Cell Longev. 2019. https://doi.org/10.1155/2019/9137654.

15. Zhang L, Deng S. Effects of astragaloside IV on inflammation and immunity in rats with experimental periodontitis. Braz Oral Res. 2019;33:e32.

16. Chang CP, Liu YF, Lin HJ, Hsu CC, Cheng BC, Liu WP, Lin MT, Hsu SF, Chang LS, Lin KC. Beneficial effect of astragaloside on Alzheimer's disease condition using cultured primary cortical cells under beta-amyloid exposure. Mol Neurobiol. 2016;53:7329-40.

17. Chan WS, Durairajan SS, Lu JH, Wang Y, Xie LX, Kum WF, Koo I, Yung KK, Li M. Neuroprotective effects of Astragaloside IV in 6-hydroxydopaminetreated primary nigral cell culture. Neurochem Int. 2009;55:414-22.

18. Du RH, Zhou Y, Xia ML, Lu M, Ding JH, Hu G. alpha-Synuclein disrupts the anti-inflammatory role of Drd2 via interfering beta-arrestin2-TAB1 interaction in astrocytes. J Neuroinflammation. 2018;15:258.

19. Nakamura S, Oba M, Suzuki M, Takahashi A, Yamamuro T, Fujiwara M, Ikenaka K, Minami S, Tabata N, Yamamoto K, et al. Suppression of autophagic activity by Rubicon is a signature of aging. Nat Commun. 2019; 10:847.

20. Killilea DW, Atamna H, Liao C, Ames BN. Iron accumulation during cellular senescence in human fibroblasts in vitro. Antioxid Redox Signal. 2003;5:507-16.

21. Salminen A, Ojala J, Kaarniranta K, Haapasalo A, Hiltunen M, Soininen H. Astrocytes in the aging brain express characteristics of senescenceassociated secretory phenotype. Eur J Neurosci. 2011;34:3-11.

22. Bhat $R$, Crowe EP, Bitto A, Moh M, Katsetos CD, Garcia FU, Johnson FB, Trojanowski JQ, Sell C, Torres C. Astrocyte senescence as a component of Alzheimer's disease. PLoS One. 2012;7:e45069.

23. Turnquist C, Beck JA, Horikawa I, Obiorah IE, Von Muhlinen N, Vojtesek B, Lane DP, Grunseich C, Chahine JJ, Ames HM, et al. Radiation-induced astrocyte senescence is rescued by Delta133p53. Neuro-Oncology. 2019;21: 474-85.

24. Cao P, Zhang J, Huang Y, Fang Y, Lyu J, Shen Y. The age-related changes and differences in energy metabolism and glutamate-glutamine recycling in the d-gal-induced and naturally occurring senescent astrocytes in vitro. Exp Gerontol. 2019;118:9-18.

25. Bitto A, Sell C, Crowe E, Lorenzini A, Malaguti M, Hrelia S, Torres C. Stressinduced senescence in human and rodent astrocytes. Exp Cell Res. 2010; 316:2961-8.

26. Jiang $Y$, Dong G, Song Y. Nucleus pulposus cell senescence is alleviated by resveratrol through regulating the ROS/NF-kappaB pathway under highmagnitude compression. Biosci Rep. 2018. https://doi.org/10.1042/ BSR20180670.

27. Jin J, Richardson L, Sheller-Miller S, Zhong N, Menon R. Oxidative stress induces p38MAPK-dependent senescence in the feto-maternal interface cells. Placenta. 2018;67:15-23.

28. Vasileiou P, Evangelou K, Vlasis K, Fildisis G, Panayiotidis MI, Chronopoulos E, Passias PG, Kouloukoussa M, Gorgoulis VG, Havaki S. Mitochondrial homeostasis and cellular senescence. Cells. 2019. https://doi.org/10.3390/ cells8070686.

29. Hernandez-Segura A, Nehme J, Demaria M. Hallmarks of cellular senescence. Trends Cell Biol. 2018;28:436-53.

30. Garcia-Prat L, Martinez-Vicente M, Perdiquero E, Ortet L, Rodriguez-Ubreva J, Rebollo E, Ruiz-Bonilla V, Gutarra S, Ballestar E, Serrano AL, et al. Autophagy maintains stemness by preventing senescence. Nature. 2016;529:37-42.

31. Hansen M, Rubinsztein DC, Walker DW. Autophagy as a promoter of longevity: insights from model organisms. Nat Rev Mol Cell Biol. 2018;19:579-93.

32. Rivero-Rios P, Madero-Perez J, Fernandez B, Hilfiker S. Targeting the autophagy/lysosomal degradation pathway in Parkinson's disease. Curr Neuropharmacol. 2016;14:238-49.

33. Lee DE, Bareja A, Bartlett DB, White JP. Autophagy as a therapeutic target to enhance aged muscle regeneration. Cells. 2019. https://doi.org/10.3390/ cells8020183.

34. Garcia-Prat L, Munoz-Canoves P, Martinez-Vicente M. Dysfunctional autophagy is a driver of muscle stem cell functional decline with aging. Autophagy. 2016;12:612-3.

35. Fivenson EM, Lautrup S, Sun N, Scheibye-Knudsen M, Stevnsner T, Nilsen H, Bohr VA, Fang EF. Mitophagy in neurodegeneration and aging. Neurochem Int. 2017;109:202-9.
36. Araya J, Tsubouchi K, Sato N, Ito S, Minagawa S, Hara H, Hosaka Y, Ichikawa A, Saito N, Kadota T, et al. PRKN-regulated mitophagy and cellular senescence during COPD pathogenesis. Autophagy. 2019;15:510-26.

37. Hou J, Cui C, Kim S, Sung C, Choi C. Ginsenoside F1 suppresses astrocytic senescence-associated secretory phenotype. Chem Biol Interact. 2018;283:75-83.

38. Yu C, Narasipura SD, Richards MH, Hu XT, Yamamoto B, Al-Harthi L. HIV and drug abuse mediate astrocyte senescence in a beta-catenin-dependent manner leading to neuronal toxicity. Aging Cell. 2017;16:956-65.

39. Booth $H$, Hirst WD, Wade-Martins R. The role of astrocyte dysfunction in Parkinson's disease pathogenesis. Trends Neurosci. 2017;40:358-70.

40. Gorshkov K, Aguisanda F, Thorne N, Zheng W. Astrocytes as targets for drug discovery. Drug Discov Today. 2018;23:673-80

41. Clarke LE, Liddelow SA, Chakraborty C, Munch AE, Heiman M, Barres BA. Normal aging induces A1-like astrocyte reactivity. Proc Natl Acad Sci U S A. 2018:115:E1896-905.

42. Yun SP, Kam TI, Panicker N, Kim S, Oh Y, Park JS, Kwon SH, Park YJ, Karuppagounder SS, Park $\mathrm{H}$, et al. Block of A1 astrocyte conversion by microglia is neuroprotective in models of Parkinson's disease. Nat Med. 2018;24:931-8.

43. Das MM, Svendsen CN. Astrocytes show reduced support of motor neurons with aging that is accelerated in a rodent model of ALS. Neurobiol Aging 2015:36:1130-9.

44. Crowe EP, Tuzer F, Gregory BD, Donahue G, Gosai SJ, Cohen J, Leung YY, Yetkin E, Nativio R, Wang LS, et al. Changes in the transcriptome of human astrocytes accompanying oxidative stress-induced senescence. Front Aging Neurosci. 2016;8:208

\section{Publisher's Note}

Springer Nature remains neutral with regard to jurisdictional claims in published maps and institutional affiliations.

Ready to submit your research? Choose BMC and benefit from:

- fast, convenient online submission

- thorough peer review by experienced researchers in your field

- rapid publication on acceptance

- support for research data, including large and complex data types

- gold Open Access which fosters wider collaboration and increased citations

- maximum visibility for your research: over $100 \mathrm{M}$ website views per year

At $\mathrm{BMC}$, research is always in progress.

Learn more biomedcentral.com/submissions 